\title{
Anabases
}

ANABASES Traditions et réceptions de l'Antiquité

19 | 2014

Varia

\section{Katherine HARLOE et Neville MORLET (ed.), Thucydides and the Modern World. Reception, Reinterpretation and Influence from the Renaissance to the Present}

Gaëlle Delemestre

\section{OpenEdition}

\section{Journals}

Édition électronique

URL : http://journals.openedition.org/anabases/4738

DOI : $10.4000 /$ anabases. 4738

ISSN : 2256-9421

Éditeur

E.R.A.S.M.E.

Édition imprimée

Date de publication : 1 avril 2014

Pagination : 349-351

ISSN : 1774-4296

\section{Référence électronique}

Gaëlle Delemestre, « Katherine harloe et Neville morlet (ed.), Thucydides and the Modern World

Reception, Reinterpretation and Influence from the Renaissance to the Present », Anabases [En ligne], 19

2014, mis en ligne le 01 avril 2014, consulté le 22 septembre 2020. URL : http://

journals.openedition.org/anabases/4738; DOI : https://doi.org/10.4000/anabases.4738

Ce document a été généré automatiquement le 22 septembre 2020

(c) Anabases 


\title{
Katherine HARLOE et Neville MORLET (ed.), Thucydides and the Modern World. Reception, Reinterpretation and Influence from the Renaissance to the Present
}

\author{
Gaëlle Delemestre
}

\section{RÉFÉRENCE}

Katherine HARLOE et Neville MORLET (ed.), Thucydides and the Modern World. Reception, Reinterpretation and Influence from the Renaissance to the Present, Cambridge, Cambridge University Press, 2012, 256 p. 62 livres / ISBN 978-1-107-01920-1

1 Le chapitre introductif à l'ouvrage, composé par K. Harloe et N. Morlet, rappelle la place assez mineure faite à Thucydide dans la pensée occidentale jusqu'à la traduction latine de la guerre du Péloponnèse au xve siècle, puis celle de Hobbes, en 1629, qui semble être à l'origine de l'enseignement aujourd'hui couramment reçu d'un ouvrage présentant pour la première fois une analyse réaliste de la politique et de la société. Les auteurs notent cependant l'ambiguïté d'une telle présentation, courante en Relations Internationales, quand Thucydide peut aussi être décrit par les autres sciences humaines comme militaire stratège, historiographe, reporter contre la guerre, ou philosophe et artiste réaliste. L'enjeu de l'ouvrage est de présenter cette polyvalence sans diluer le texte de Thucydide.

2 K. Hoekstra étudie les différents recours faits à Thucydide, sur la période du XvI et du XVII ${ }^{\mathrm{e}}$ siècle, pour justifier ou légitimer le recours à la guerre. L'une des lignes de son exposé est que Thucydide a été utilisé comme une autorité pour légitimer les guerres 
non seulement défensives, mais aussi préventives, sans réelle identification d'une limite à apporter au sentiment d'être en danger. Reprenant les discours successifs d'hommes politiques (Simon Renard, Henry Wotton, Paolo Scapi) ou de théoriciens (Lipsius, Gentili, Grotius, Bacon, Hobbes), il souligne les références contradictoires qui sont faites à La guerre du Péloponnèse, tantôt utilisé pour prôner l'injustice des assauts préventifs, tantôt pour illustrer à l'inverse les risques encourus à retarder le déclenchement des hostilités. L'arrière-fond théorique qui se dégage cependant du recours à Thucydide est l'idée d'une incertitude fondamentale des intentions des différentes nations les unes envers les autres, où les déséquilibres de puissance peuvent à tout moment se transformer en dangers réels pour les voisins. Thucydide devient alors le précurseur des thèses hobbesiennes d'un espace international par nature belliqueux et instable.

3 En suivant l'utilisation de Thucydide faite par les Jacobins et les Thermidoriens, N. Urbinati montre, quant à elle, qu'Athènes a été utilisée à la fois pour mettre à distance la démocratie directe de la république libérale moderne et pour mettre en garde contre les dangers immanents à la démocratie. Si les premiers utilisent ainsi Sparte pour défendre l'avantage de la stabilité sur la démocratie, associée à l'anarchie, le manque de vertu ou l'instabilité des factions, les seconds, recourant à Thucydide, vont la critiquer pour son irrationalité, le manque de compétence et la tyrannie de la majorité qu'elle favorise. Cette mise en relation dualiste du passé et de l'époque moderne illustre la réorientation de l'histoire athénienne que le mouvement révolutionnaire et postrévolutionnaire introduit en s'appuyant encore, en grande partie, sur les sources historiques données par le récit de Thucydide.

4 J. Süssmann rend compte de l'historisation du texte thucydidéen opérée dans l'Allemagne du XIXe siècle. Les historiens (Troeltsch, Niebuhr, Ranke) commencent à reconstruire l'histoire dans un sens généalogique en évoluant du compte rendu des faits vers la reconstruction analytique des événements. Thucydide n'est alors plus considéré comme fournissant des arguments à une certaine idéologie, mais comme une source d'enseignement sur les phénomènes qui ont agité le monde hellénique pendant la guerre du Péloponnèse. Il devient digne d'un intérêt historique, sans permettre d'être utilisé à des fins politiques. N. Morley expose quant à lui les analyses faites par Wilhelm Roscher de Thucydide, en qui il voit présentes à la fois la rigueur scientifique Thucydide ne s'arrêtant pas aux croyances de ses contemporains, cherchant l'impartialité - et la grandeur artistique, dans le fait d'avoir permis à son intelligence de répondre face à l'expérience.

Deux figures-clés du xix siècle anglais, J.S. Mill et Matthew Arnold, retrouvent en Thucydide une source de réflexion pour aborder l'évolution de la société britannique. E. Potter revient sur les temps forts de leur œuvre où Athènes et Thucydide sont l'objet d'une analyse détaillée. C'est la tension inhérente à Athènes, entre la grandeur d'une cité capable de parvenir à un tel degré de savoir, de beauté et de progrès politique, et l'instabilité d'un régime conduit sous la pression de la guerre à prendre de mauvaises décisions, qui les amène à pointer les paradoxes du système démocratique et à envisager les moyens permettant d'en contrer les effets négatifs.

6 La guerre du Péloponnèse a rapidement été accessible en anglais, français et allemand. En le traduisant en grec, E. Venizelos, homme politique éminent de la première moitié du $\mathrm{xx}^{\mathrm{e}}$ siècle, engage un parallèle, favorisé par ses choix de traduction, entre la situation hellénique décrite par Thucydide et la situation de la Grèce défaite après la campagne 
en Asie Mineure, en 1919-1922. Telle est du moins la démonstration faite par E. Greenwood dans son écrit. Actualisant les propos de Thucydide, il en fait l'observateur du premier schisme national et conduit par là ses contemporains à cesser les querelles intestines qui paralysent la Grèce. Ainsi sont dégagées des lois qui tracent entre les différents moments de l'Histoire comme une continuité, une certaine circularité du réel que l'on pourrait rompre en prêtant plus d'attention aux leçons qu'elle nous prodigue.

7 La contribution de S. Forde consiste à estimer en quel sens Thucydide peut effectivement être considéré comme un réaliste, comme le soutient le courant réaliste en Relations Internationales, et comme l'avance $\mathrm{H}$. Morgenthau. Il cherche en conséquence à trouver, dans l'œuvre de cet auteur ainsi que chez Machiavel et Hobbes, eux aussi considérés comme "réalistes ", les motivations réelles données pour justifier les guerres. Il observe que les trois sources fondamentales de conflit entre nations, pour ces trois auteurs, sont la peur de la puissance adverse, l'intérêt et l'honneur. Il en déduit que ces théoriciens ne peuvent prendre le nom de réalistes au sens contemporain, Morgenthau ayant sous-estimé l'importance du sentiment d'insécurité et la passion de l'ambition. Les trois motifs des guerres internationales diagnostiqués n'ont pas la rationalité que Morgenthau décrit comme étant à leur base.

8 R. Ned Lebow considère aussi la relation entre Thucydide et les Relations Internationales, ces dernières ayant connu un fort développement depuis la fin de la guerre froide, avec un besoin croissant de retour aux textes fondateurs. Trois raisons expliquent la recrudescence des études classiques: la justification de ses propres arguments, la délégitimisation de certaines lectures, et la revendication d'une certaine tradition interprétative.

\section{AUTEURS}

\section{GAËLLE DELEMESTRE}

CERSES, UMR 8137 CNRS/ Université Paris-Descartes

gdelemestre@gmail.com 